\title{
On the Design of a Web-based Decision Support System for Brain Tumour Diagnosis using Distributed Agents
}

\begin{tabular}{|c|c|c|c|}
\hline $\begin{array}{l}\text { Carles Arús } \\
\text { U.A. Barcelona }\end{array}$ & $\begin{array}{l}\text { Bernardo Celda } \\
\text { U. València }\end{array}$ & $\begin{array}{l}\text { dan Dasmahaptra } \\
\text { Southampton }\end{array}$ & $\begin{array}{l}\text { David Dupplaw } \\
\text { U. Southampton }\end{array}$ \\
\hline $\begin{array}{c}\text { Horacio González-Vélez } \\
\text { U. Edinburgh }\end{array}$ & $\begin{array}{l}\text { Sabine Van Huffel } \\
\text { KU Leuven }\end{array}$ & $\begin{array}{c}\text { Paul Lewis } \\
\text { U. Southampton }\end{array}$ & $\begin{array}{l}\text { Magí Lluch i Ariet } \\
\text { MicroArt }\end{array}$ \\
\hline $\begin{array}{l}\text { Mariola Mies } \\
\text { MicroArt \& U. Edi }\end{array}$ & $\begin{array}{r}\text { And } \\
\text { U. Birmin }\end{array}$ & $\begin{array}{l}\text { Peet } \\
\text { am \& BCH }\end{array}$ & $\begin{array}{l}\text { tserrat Robles } \\
\text { ITACA }\end{array}$ \\
\hline
\end{tabular}

\section{Abstract}

This paper introduces HealthAgents, an EC-funded research project to improve the classification of brain tumours through multi-agent decision support over a distributed network of local databases or Data Marts. HealthAgents will not only develop new pattern recognition methods for a distributed classification and analysis of in vivo MRS and ex vivo/in vitro HRMAS and DNA data, but also define a method to assess the quality and usability of a new candidate local database containing a set of new cases, based on a compatibility score.

\section{Introduction}

Brain tumours remain an important cause of morbidity and mortality in Europe. Diagnosis using Magnetic Resonance Imaging (MRI) is non-invasive, but only achieves 60-90\% accuracy depending on the tumour type and grade. The current gold standard classification of brain tumours by biopsy and histopathological analysis involves invasive surgical procedure and incurs a risk.

Nowadays the diagnosis and treatment of brain tumours is typically based on clinical symptoms, radiological appearance and often a histopathological diagnosis of a biopsy. However, treatment response of histologically or radiologically-similar tumours can vary widely, particularly in children. Magnetic Resonance Spectroscopy (MRS) is a non-invasive technique for determining the tissue biochemical composition (metabolomic profile) of a tumour. Additionally, the genomic profile, determined using DNA microarrays, facilitates the classification of tumour grades and types not trivially distinguished by morphologic appearance.
Thus, the HealthAgents project [13] entails the development of a web-based decision support system (DSS) which employs MRS and genomic profiles. This DSS will deploy an agent-based architecture in order to provide a distributed diagnostic tool for brain tumours, implement data mining techniques, transfer clinical data and extract information. The distributed nature of our approach will help the users to observe local centre policies for sharing information whilst allowing them to benefit from the use of a distributed data warehouse (d-DWH). Moreover, it will permit the design of local classifiers targeting a specific patient population.

We argue that this new information for classifying tumours along with clinical data, should be securely and easily accessible in order to improve the diagnosis and prognosis of tumours. All data will be stored anonymously, and securely through a network of data marts based on all this information acquired and stored at centres throughout $\mathrm{Eu}-$ rope. This network will grant bona-fide access to an organisation in return for its contribution of clinical data to a d-DWH/Decision Support System (d-DSS).

The rest of this paper is structured as follows. First, we provide some background on the underlying technologies for this project: brain tumour detection and agent technology. Then we provide the architectural specification. Finally, we conclude with our future work.

\section{Background}

\subsection{Brain Tumour Diagnosis}

Brain tumours remain an important cause of morbidity and mortality and afflict an increasing percentage of aging adults with a crude incidence rate of 8 per 100,000 inhabitants in Europe [2]. In children over 1 year of age, brain tumours are the most common solid malignancies that cause 

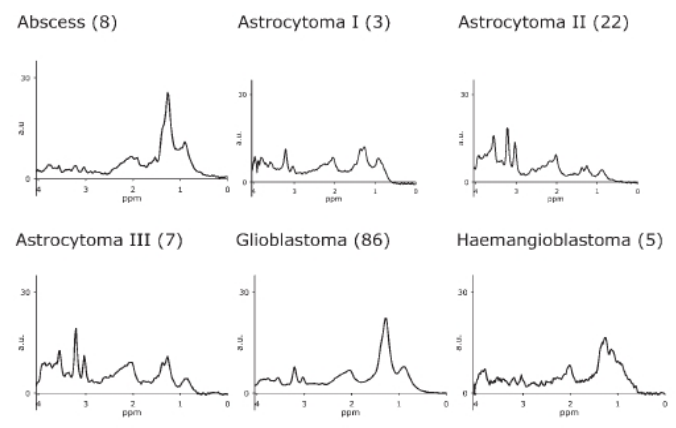

Lymphoma (10) Meningioma (58) Metastasis (38)

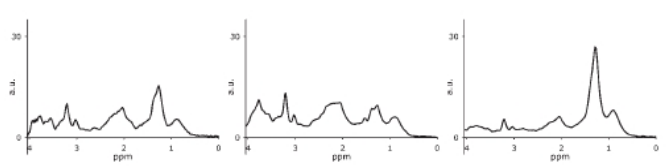

Normal brain (22) Oligoastroctoma II (6) Oligodendroglioma II (7)

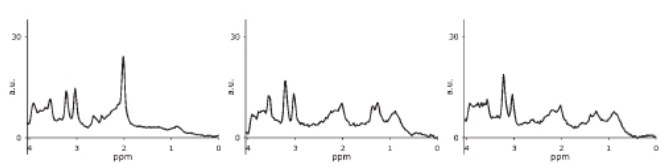

PNET (9) Schwannoma (4)

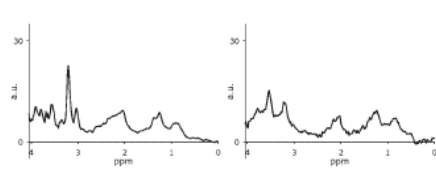

Figure 1. Mean short echo spectra of representative pathologies in a validated-DB from the INTERPRET project [6]. These were obtained by averaging spectra normalised to the Euclidian norm. The vertical axis is displayed in the same arbitrary units (a.u.) scale for all types. The horizontal axis labels ppm values. Number of cases of each type in parentheses. The most relevant metabolites are: lipids, 0.9 and $1.29 \mathrm{ppm}$; $\mathrm{N}$-acetyl-containing compounds, $2.03 \mathrm{ppm}$; acetate, $1.9 \mathrm{ppm}$; macromolecules and glutamate/glutamine containing compounds, 2-2.5 ppm; creatine, $3.03 \mathrm{ppm}$; choline-containing compounds, $3.21 \mathrm{ppm}$; myo-inositol and glycine, $3.55 \mathrm{ppm}$; glutamate/glutaminecontaining compounds and alanine, $3.77 \mathrm{ppm}$. (Adapted from Figure 4 of [12] (c)2006 John Wiley \& Sons Limited. Reproduced with permission.)

disease-related death.

Diagnosis using Magnetic Resonance Imaging (MRI) is non-invasive, but only achieves 60-90\% accuracy depending on the tumour type and grade [5]. The current gold standard classification of a brain tumour by histopathological analysis of biopsy is an invasive surgical procedure and incurs a risk of 2.4-3.5\% morbidity and $0.2-0.8 \%$ mortality, in addition to healthcare costs and stress to patients. For tumours that evolve slowly (e.g. pilocytic astrocytoma in children), repeated biopsies may not be advisable or practical.
There is a need to improve brain tumour classification, and to provide non-invasive methods for brain tumour diagnosis and prognosis, to aid patient management and treatment. Three techniques are available to address these needs:

1. Magnetic Resonance Spectroscopy (MRS) [4] is a noninvasive technique that provides biochemical information on tissue in vivo.

2. HR-MAS $[8,1]$ is applied to biopsies in vitro in order to improve characterisation. Also, DNA microarray analysis of biopsies can determine tumour phenotype from gene expression profiles and predict better survival than classical histology $[11,10]$.

3. MRS, coupled with conventional MRI, provides metabolite profiles of a single voxel (SV) of tumour tissue $[12,4]$ (see Fig. 1). It also produces a molecular image of particular tumour metabolites in 10 minutes using multi-voxel (MV) techniques (Fig. 2).

\section{$2.2 \quad$ Agent technology}

Several modern complex distributed systems are composed of customisable building blocks, known as agents. Surveys on agent technology enumerate four important characteristics of agent technology [3]. First, agents possess an internal knowledge-based state that can be dynamically altered. Second, they have dynamic reasoning capabilities that determine their internal behaviour through constraints or goals. Third, they sustain a communication status that enables them to interact with agents or human entities. Last, they feature a unique identity that provides roaming and service advertising capabilities.

Extensive research in agent systems has been conducted in Europe, as evidenced by the reach of the AgentLink membership [15]. Data mining agents present human researchers with a set of potential hypotheses deduced from the data sources. Thus, with the information explosion caused by genomics and proteomics research, there is a great need for automated information-gathering agents in order to assist human researchers conducting automated or semi-automated testing of data.

Recent initiatives have introduced the use of agents and web services to genome analysis and decision support in the biomedical sciences [9]. On the other hand, the design of web-based support has evolved into a mature research field for the integration of domain specific studies with computer science [16]. However, scant multi-disciplinary research has been channelled to the distributed bioinformatics domain, where numerous databases and analysis tools are independently administered in geographically distinct localities, lending themselves almost ideally to the adoption of a distributed intelligent multi-agent approach with enhanced multi-layer security and a web-based user interface. 


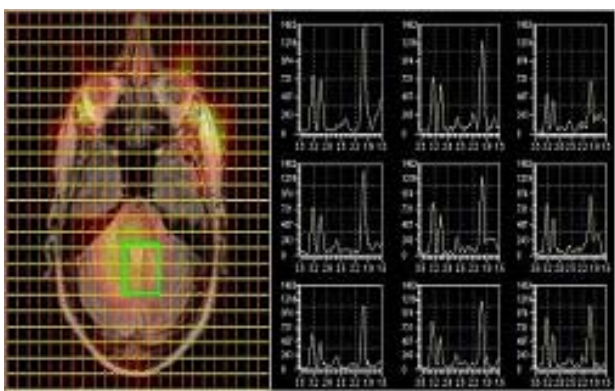

(a)

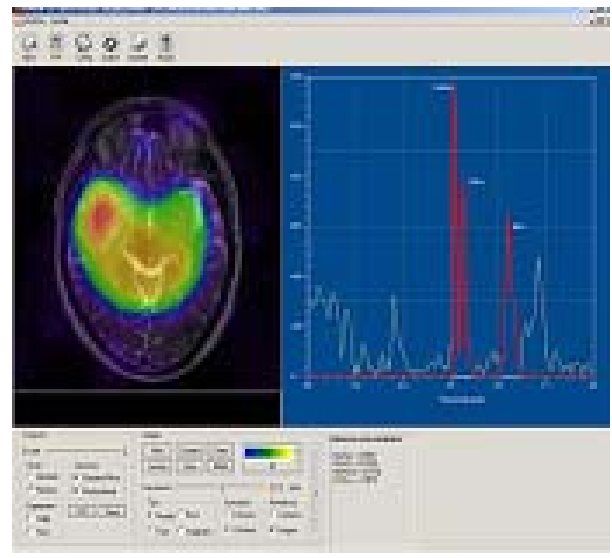

(b)

Figure 2. (a) Molecular image of Cho (choline) concentration distribution from CSI spectra of a patient with a Glioblastoma (red indicates highest Cho levels in the tumour) including the deconvoluted spectrum. (b) CSI spectra from the rear cavity with a demyelisation lesion. The nine spectra shown, from the selected green square, present the most abnormal region bottom right (Images generated using SIView 2.0 [7])

\section{Architectural specification}

By focusing on brain tumour diagnosis and prognosis, the HealthAgents project is to apply agent technology to securely connect user sites with a distributed database. It will employ agent negotiation and argumentation mechanisms developed for distributed resource allocation problems.

Moreover, HealthAgents intends to build a completely distributed repository with local databases. Grid technologies such as multi-site data partition and distributed data sharing will permit the seamless access to different databases across sites.

We argue that a d-DSS will furnish a completely new approach to brain tumour diagnosis. Since inferences from local predictions may well conflict with one another, reasoned argument between intelligent agents, acting on behalf

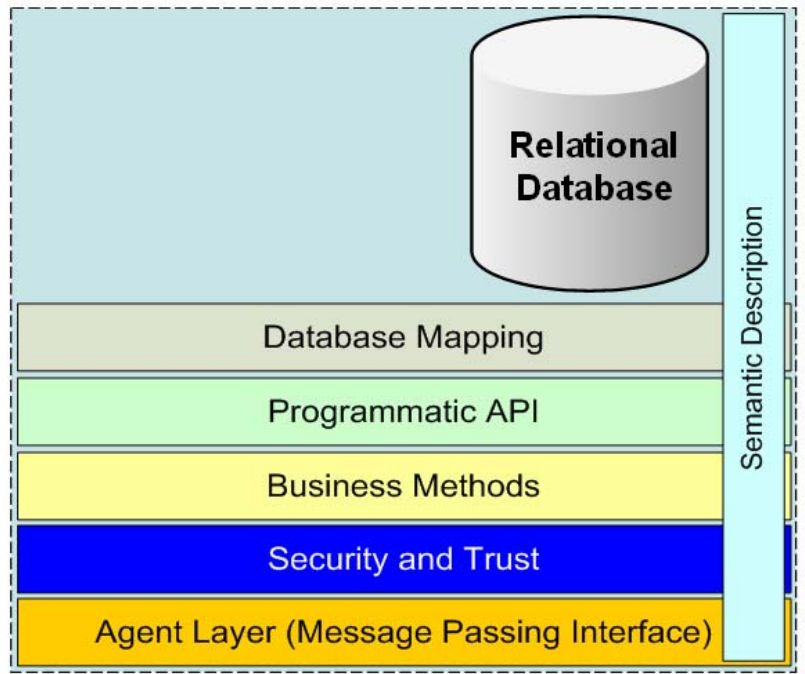

Figure 3. HealthAgents multi-layer framework)

of scientists, in a multi-agent system, will foster consensus.

The HealthAgents project intends not only to apply agent technology to the biomedical field in a multi-disciplinary fashion, but also develop the first distributed repository for brain tumour diagnosis, leading eventually to the formation of a special interest data grid.

A centralised DSS is already available from the INTERPRET project $[14,6]$ to facilitate the clinical use of MRS in brain tumour diagnosis which uses a classification based on histopathological diagnosis. A more elaborated DSS, combining MRS biochemical profiles from Single Voxel (SV) and metabolic spatial distribution by Chemical Shift Imaging (CSI) MRS in vivo data, is currently being developed and implemented in the eTUMOUR project [6]. The eTUMOUR DSS will eventually improve and facilitate the clinical application of MRS in adults and paediatric brain tumour diagnosis, prognosis and treatment selection using a classification based on the combination of histology results and high resolution metabolic profiles (HR-MAS) and transcriptomic (DNA micro-arrays) ex vivo data.

The HealthAgents d-DSS will build upon these projects and include additional MRS data, such as childhood tumours and less common adult tumours, using new classifications based on genetics. The development of this new d-DWH (the "d-DSS"), incorporating concepts of networking, agent technology, and data mining, must increase the number of accessible cases, yielding to an improved classifier, that in turn will achieve the goals described in this proposal.

HealthAgents proposes a multi-layer system architecture 
as depicted in Fig. 3. The database-mapping layer is used to map a relational database schema to the HealthAgents ontological schema. The programming API layer abstracts the underlying database interaction from the agent architecture. The business methods layer contributes to the main control flow of an agent such as the new case classification, new classifiers reception, and data retrieval.

The security and trust layer is a crucial system component due to the sensitivity of the data. Its functionalities are access control, data marshalling, tracking of on-going data, and the evaluation of reputation and trust of agents. The agent layer is in charge of all the communications and allows their abstraction from the rest of the system to allow flexibility in the underlying framework. The semantic description layer will contain the description of what the agent holds and what it is able to do.

\section{Conclusions}

In vivo MRS combined with in vitro MAS and gene expression promises to improve the classification of brain tumours and yield novel biomarkers for prognosis. Considerable amounts of highly complex data are required to build reliable specific tumour classifiers and it is a challenge to collect and manage this data. HealthAgents will address this problem by building a distributed system of databases centred on the users and managed by agents. As a result, HealthAgents proposes a unique blend of state-of-the-art technologies to develop novel clinical tools for the diagnosis, management and understanding of brain tumours.

We have introduced the HealthAgents project, its objectives, and scope. Although tangible results are to be produced soon, we strongly believe that the conditions are given to produce an innovative software system to help in the fight against one of the most pernicious diseases of our time: cancer.

\section{Acknowledgement}

This research is supported by the EC under the HealthAgents project (STREP contract no.: IST-2004-27214).

\section{References}

[1] S. Barton, F. Howe, A. Tomlins, S. Cudlip, J. Nicholson, B. Bell, and J. Griffiths. Comparison of in vivo $1 \mathrm{H}$ MRS of human brain tumours with 1H HR-MAS spectroscopy of intact biopsy samples in vitro. Magn. Reson. Mat. Phys. Biol. Med., 8(2):121-128, 1999.

[2] F. Bray, R. Sankila, J. Ferlay, and D. M. Parkin. Estimates of cancer incidence and mortality in Europe in 1995. Eur. $J$. Cancer, 38(1):99-166, 2002.
[3] D. Brugali and K. Sycara. Towards agent oriented application frameworks. ACM Computing Surv., 32(1):21-27, 2000.

[4] F. A. Howe and K. S. Opstad. 1H MR spectroscopy of brain tumours and masses. NMR Biomed., 16(3):123-131, 2003.

[5] M. Julià-Sapé, D. Acosta, C. Majós, A. Moreno-Torres, P. Wesseling, J. J. Acebes, J. R. Griffiths, and C. Arús. Comparison between neuroimaging classifications and histopathological diagnoses using an international multicenter brain tumor magnetic resonance imaging database. $J$. Neurosurg., 105(1):6-14, 2006.

[6] M. Julià-Sapé, D. Acosta, M. Mier, C. Arús, D. Watson, and The INTERPRET consortium. A multi-centre, webaccessible and quality control-checked database of in vivo MR spectra of brain tumour patients. Magn. Reson. Mat. Phys. Biol. Med., 19(1):22-33, 2006.

[7] J. V. Manjón-Herrera, M. C. Martínez-Bisbal, B. Celda, L. Martí-Bonmatí, and M. Robles. SIView 2.0: A new MR spectroscopy imaging tool. Eur. Radiol. (Suppl.), 14(2):300, 2004.

[8] M. C. Martínez-Bisbal, L. Martí-Bonmatí, J. Piquer, A. Revert, P. Ferrer, J. L. Llácer, M. Piotto, O. Assemat, and B. Celda. $1 \mathrm{H}$ and 13C HR-MAS spectroscopy of intact biopsy samples ex vivo and in vivo. NMR Biomed., 17(4):191-205, 2004.

[9] E. Merelli, G. Armano, N. Cannata, F. Corradini, M. d Inverno, A. Doms, P. Lord, A. Martin, L. Milanesi, S. Möller, M. Schroeder, , and M. Luck. Agents in bioinformatics, computational and systems biology. Brief. Bioinform., 2006. To appear: doi : 10.1093/bib/bblo14.

[10] P. Mischel, T. Cloughesy, and S. Nelson. DNA-microarray analysis of brain cancer: molecular classification for therapy. Nature Rev. Neuroscience, 5:782-792, 2004.

[11] C. L. Nutt, D. R. Mani, R. A. Betensky, P. Tamayo, J. G. Cairncross, C. Ladd, U. Pohl, C. Hartmann, M. E. McLaughlin, T. T. Batchelor, P. M. Black, A. von Deimling, S. L. Pomeroy, T. R. Golub, and D. N. Louis. Gene expressionbased classification of malignant gliomas correlates better with survival than histological classification. Cancer Res., 63:1602-1607, 2003.

[12] A. R. Tate, J. Underwood, D. M. Acosta, M. Julià-Sapé, C. Majós, A. Moreno-Torres, F. A. Howe, M. van der Graaf, V. Lefournier, M. M. Murphy, A. Loosemore, C. Ladroue, P. Wesseling, J. L. Bosson, M. E. C. nas, A. W. Simonetti, W. Gajewicz, J. Calvar, A. Capdevila, P. R. Wilkins, B. A. Bell, C. Rémy, A. Heerschap, D. Watson, J. R. Griffiths, and C. Arús. Development of a decision support system for diagnosis and grading of brain tumours using in vivo magnetic resonance single voxel spectra. NMR Biomed., 19(4):411434, 2006.

[13] The HealthAgents Consortium. HealthAgents. http:// groups. inf .ed.ac.uk/HealthAgents, 2006. ECfunded Specific Targeted Research Project Contract no.: IST2004-27214.

[14] Universitat Autònoma de Barcelona. INTERPRET project. http: //azizu.uab.es/INTERPRET/, 2005.

[15] University of Southampton. AgentLink. http://www. agentlink.org/, 2005.

[16] J. T. Yao. Design of web-based support systems. In 8th Int Conf on Computer Science and Informatics, pages 349-352, Salt Lake City, USA, July 2005. CSI. 